\title{
A Multi-Relaxation-Time Lattice Boltzmann Method on Non- Uniform Grids for Large Eddy Simulation of Rayleigh-Bénard Convection Using Two Sub-Grid Scale Models
}

\author{
A. R. Rahmati ${ }^{1 \dagger}$, M. Ashrafizaadeh ${ }^{2}$ and E. Shirani ${ }^{3}$ \\ ${ }^{l}$ Department of Mechanical Engineering, Faculty of Engineering, University of Kashan, Kashan, Iran \\ ${ }^{2}$ Department of Mechanical Engineering, Isfahan University of Technology, Isfahan, Iran \\ ${ }^{3}$ Foolad Institute of Technology, Fooladshahr, Isfahan, 8491663763, Iran
}

†Corresponding Author Email: ar_rahmati@kashanu.ac.ir

(Received October 22, 2012; accepted May 1, 2013)

\begin{abstract}
In the present work, for the first time, the application of a Multi-Relaxation-Time Lattice Boltzmann (MRT-LB) model for large-eddy simulation (LES) of turbulent thermally driven flows on non-uniform grids is considered. A Taylor series expansion and Least square based Lattice Boltzamnn method (TLLBM) has been implemented in order to use a nonuniform mesh. It permits to reduce the required mesh size and consequently the computational cost to simulate the turbulent buoyant flow fields. The implementation is discussed in the context of a MRT-LB model in conjunction with both Smagorinsky and mixed scale viscosity sub-grid models. At first, to validate the code, a multi-relaxation-time lattice Boltmann method on non-uniform grid is utilized to simulate a lid-driven cavity flow. Then large eddy simulation of this model is applied to simulate a turbulent Rayleigh-Bénard convection at different Rayleigh numbers in ranging $10^{4}$ to $10^{15}$ for Prantdl number of 0.71 . The simulation results show that lattice Boltzmann method is capable to simulate turbulent convection flow problems at high Rayleigh numbers.
\end{abstract}

Keywords: Lattice Boltzmann method, MRT-LBM, LES, TLLBM.

\section{NOMENCLATURE}

b total number of lattice streaming vectors

c magnitude of lattice streaming vectors

$c_{s} \quad$ speed of sound

$C_{S} \quad$ Smagorinsky constant

D dimension of system

$\mathbf{e}_{\alpha} \quad$ lattice streaming vector in $\alpha$ direction

$e \quad$ kinetic energy

$f_{\alpha} \quad$ density distribution function

$f_{\alpha}^{e q} \quad$ equilibrium density distribution function

$g \quad$ acceleration due to gravity

$L_{y} \quad$ height or vertical length

$L_{x} \quad$ horizontal length

$\mathbb{M} \quad$ moment space

$M \quad$ transformation matrix

$\begin{array}{ll}\mathbb{V} & \text { discrete velocity space } \\ \mathbf{u} & \text { flow velocity vector } \\ \hat{S} & \text { relaxation matrix } \\ \bar{S} & \text { filtered mean momentum flux } \\ S_{i j} & \text { strain rate tensor } \\ t & \text { time } \\ T & \text { temperature } \\ x, y & \text { Cartesian coordinate system } \\ \text { Greek } & \text { symbols } \\ \beta & \text { thermal expansion coefficient } \\ \delta r & \text { lattice spacing } \\ \delta t & \text { time step } \\ \varepsilon & \text { square of kinetic energy } \\ \kappa & \text { fluid thermal diffusivity }\end{array}$


A.R. Rahmati et al. / JAFM, Vol. 7, No. 1, pp. 89-102, 2014.

$\begin{array}{ll}m & \text { moment } \\ p & \text { pressure } \\ p_{i j} & \begin{array}{l}\text { second-order moments of the distribution } \\ \text { function } \\ v / \alpha, \text { Prandtl number }\end{array} \\ P r & \text { heat flux vector } \\ \mathbf{q} & \text { momentum fluxes } \\ Q_{i j} & \text { the characteristic filtered rate of strain } \\ \bar{Q} & \text { position vector } \\ \mathbf{r} & \frac{g \beta \Delta T L_{y}{ }^{3}}{v \alpha}, \text { Rayleigh number } \\ R a & \text { top lid velocity } \\ U & \mathrm{x}, \text { and y components of the flow velocity vector } \\ u, v & \end{array}$

\section{INTRODUCTION}

Thermally driven convection flow in enclosures which are heated from below (Rayleigh-Bénard convection) is an important problem that has been investigated for several decades (Siggia, 1994; Kadanoff, 2001; Kerr, 1996; Sreenivasan and Donnelly, 2001). The kineticbased lattice Boltzmann method is a powerful tool for simulating fluid flows and modeling the physics in fluids (Chen and Doolen, 1998a; Rahmati and Ashrafizaadeh, 2010). However, the application of LB model to thermal problems has not attained great success for the thermal models due to the severe numerical instability caused by breaking the isothermal condition (Lallemand and Luo, 2003). The existing approaches for creating thermal LB models can be categorized into three categories, i.e., the multi-speed lattice Boltzmann scheme(Teixeira et al., 2000), the double-density-distribution-function lattice Boltzmann (DDDFLB) approach (Chen and Doolen, 1998b), and the hybrid thermal lattice Boltzmann (HTLB) technique (Lallemand and Luo , 2003). The multi-speed scheme is a straightforward extension of the isothermal LB models, in which only the density distribution function is used; the DDDFLB approach uses two different density distribution functions, one for the velocity field and the other for the internal energy field; the HTLB technique is similar to the DDDFLB approach except that the internal energy equation is solved by finitedifference methods, rather than by solving the lattice Boltzmann method.

As mentioned above, the main difficulty of the thermal lattice Boltzmann models is their numerical instability. In the present work, for the first time, the important issues on the stability of lattice Boltzmann models have been studied and a stable combination model has been proposed and applied to simulate turbulent convective flows (Rahmati and Ashrafizaadeh, 2010).Using various thermal LB models, different numerical simulations have been performed to study 2D Rayleigh-Bénard convection

$\begin{array}{ll}\kappa_{t} & \text { turbulent heat diffusivity } \\ \rho & \text { local density } \\ \tau_{0} & \text { molecular relaxation time } \\ \tau_{t} & \text { the relaxation time corresponding to the } \\ & \text { turbulence or eddy viscosity } v_{t} \\ \tau_{\text {total }} \quad \text { effective relaxation time } \\ v_{0} \quad \text { molecular kinematic viscosity } \\ v_{t} \quad \text { turbulence or eddy viscosity } \\ v_{\text {total }} \quad \text { effective kinematic viscosity } \\ \text { Subscripts and superscripts } \\ \text { eq }\end{array}$

(Shan, 1997; Nwatchok et al., 2010; Kao and Yang, 2007). Although the results provided by these studies for low Rayleigh number are in good agreement with the CFD existing data, however, investigation about this flow at high Rayleigh numbers was not conducted. The present study employs, for the first time, HTLB model, which is based on the multi-relaxation-time lattice Boltzmann method proposed by Du et al. (2006), with the Boussinesq approximation to simulate turbulent Rayleigh-Bénard convection on a non-uniform mesh using Taylor series expansion-and least square-based Lattice Boltzamnn method (Niu et al., 2003).

\section{GOVERNING EQUATION}

\subsection{Multi-Relaxation-Time Lattice Boltzmann Method for Large Eddy simulation}

The lattice Boltzmann method utilizes a particle distribution function to describe the collective behavior of fluid molecules. In LB methods, particles are assumed to move synchronously along the bonds of a regular lattice, and satisfy the discrete form of the lattice Boltzmann equation. Perhaps, the simplest and consequently the most popular form of the lattice Boltzmann method is the Single-Relaxation-Time (SRT) lattice Boltzmann method (Chen and Doolen, 1998a). However, this simplicity comes at the expense of some deficiencies (e.g. numerical instability and inaccuracy in implementing boundary conditions). To overcome some of the SRT-LBM deficiencies, the multi-relaxation-time lattice Boltzmann method (Rahmati and Ashrafizaadeh, 2010) has been developed.

The multi-relaxation-time lattice Boltzmann equation can be written as (Chen and Doolen, 1998a; Du et al., 2006):

$$
\begin{aligned}
& \left|f\left(\mathbf{r}+\mathbf{e}_{\alpha} \delta t, t_{n}+\delta t\right)\right\rangle-\left|f\left(\mathbf{r}, t_{n}\right)\right\rangle= \\
& -M^{-1} S\left[\left|m\left(\mathbf{r}, t_{n}\right)\right\rangle-\left|m^{(e q)}\left(\mathbf{r}, t_{n}\right)\right\rangle\right],
\end{aligned}
$$


A.R. Rahmati et al. / JAFM, Vol. 7, No. 1, pp. 89-102, 2014.

where elements of the diagonal matrix $\hat{S}$ are relaxation $\operatorname{rates}\left\{s_{\alpha} \mid \alpha=1,2, \ldots,(b+1)\right\}, \quad\left|m^{(e q)}\right\rangle \quad$ is the equilibrium-moment vector and its components are the equilibria of the moments, $\left|f\left(\mathbf{r}, t_{n}\right)\right\rangle$ is the distribution function vector and $M$ is transformation matrix.

For D2Q9 model (Fig. 1), the lattice velocities are defined as:

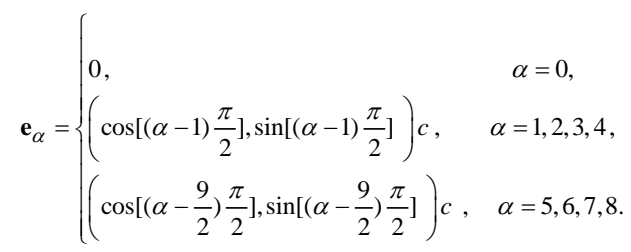

where $c$ is the particle velocity and is given by $\sqrt{3 R T_{m}}$ where $R$ is the gas constant and $T_{m}$ is average value of temperature.

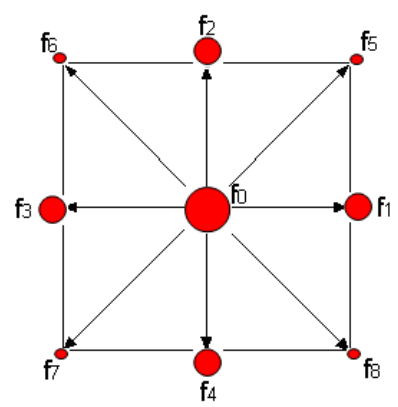

Fig. 1. Nine- particle velocity $\mathbf{e}_{\alpha}$ in the $2 \mathrm{D}$ square lattice

The transformation matrix $M$ for $\mathrm{D} 2 \mathrm{Q} 9$ model is given by:

$$
M=\left[\begin{array}{ccccccccc}
1 & 1 & 1 & 1 & 1 & 1 & 1 & 1 & 1 \\
-4 & -1 & -1 & -1 & -1 & 2 & 2 & 2 & 2 \\
4 & -2 & -2 & -2 & -2 & 1 & 1 & 1 & 1 \\
0 & 1 & 0 & -1 & 0 & 1 & -1 & -1 & 1 \\
0 & -2 & 0 & 2 & 0 & 1 & -1 & -1 & 1 \\
0 & 0 & 1 & 0 & -1 & 1 & 1 & -1 & -1 \\
0 & 0 & -2 & 0 & 2 & 1 & 1 & -1 & -1 \\
0 & 1 & -1 & 1 & -1 & 0 & 0 & 0 & 0 \\
0 & 0 & 0 & 0 & 0 & 1 & -1 & 1 & -1
\end{array}\right] .
$$

The relaxation matrix $\hat{S}$ is diagonal in the moment space $M$ :

$$
\hat{S} \equiv \operatorname{diag}\left(\begin{array}{lllllllll}
1 & s_{1} & s_{2} & 1 & s_{4} & 1 & s_{6} & s_{7} & s_{8}
\end{array}\right) .
$$

The nine moments for $\mathrm{D} 2 \mathrm{Q} 9$ model are:

$$
|m\rangle=\left(\begin{array}{lllllllll}
\rho_{0} & e & \varepsilon & u_{x} & q_{x} & u_{y} & q_{y} & p_{x x} & p_{x y}
\end{array}\right)^{T},
$$

where $\rho_{0}$ the mass density, $e$ the energy, $\varepsilon$ square of the energy, $\mathbf{u}=\left(\begin{array}{ll}u_{x} & u_{y}\end{array}\right)$ the momentum density, and $\mathbf{q}=\left(\begin{array}{ll}q_{x} & q_{y}\end{array}\right)$ is the heat flux. Also, $p_{x x}$, and $p_{x y}$ are the stresses.

The equilibria of the moments, $m^{(e q)}$, are the functions of the invariant moments, which are the mass density $\rho_{0}$ and the velocity vector $\mathbf{u}$ for athermal fluids. However, in the current method the pressure $p$ has been used instead of the mass density $\rho_{0}$, i.e., $m^{(e q)}\left(\mathbf{r}, t_{n}\right)=m^{(e q)}\left(p\left(\mathbf{r}, t_{n}\right), u\left(\mathbf{r}, t_{n}\right)\right)$. For the D2Q9 model, the equilibria for the non-invariant moments are given by:

$$
\begin{aligned}
& e^{(e q)}=6 p+3\left(u_{x} \cdot u_{x}+u_{y} \cdot u_{y}\right), \\
& \varepsilon^{(e q)}=-9 p-3\left(u_{x} \cdot u_{x}+u_{y} \cdot u_{y}\right), \\
& q_{x}^{(e q)}=-u_{x}, \\
& q_{y}^{(e q)}=-u_{y}, \\
& p_{x x}^{(e q)}=\frac{1}{3}\left(u_{x}^{2}-u_{y}^{2}\right), \\
& p_{x y}^{(e q)}=\frac{1}{3} u_{x} u_{y},
\end{aligned}
$$

The kinematic viscosity $v$ of the model is given by:

$$
v=\frac{1}{3}\left(\frac{1}{s_{8}}-\frac{1}{2}\right)=\frac{1}{3}\left(\frac{1}{s_{7}}-\frac{1}{2}\right),
$$

\subsection{Multi-Relaxation-Time Lattice Boltzmann Method for Large Eddy simulation}

Large eddy simulation of multi-relaxation -time lattice Boltzmann method results in the filtered form of the MRT-LB method (Krafczyk et al., 2003; Van Treeck et $a l ., 2006)$ and it is given bellow:

$$
\begin{aligned}
& \left|\bar{f}\left(\mathbf{r}+\mathbf{e}_{\alpha} \delta t, t_{n}+\delta t\right)\right\rangle-\left|\bar{f}\left(\mathbf{r}, t_{n}\right)\right\rangle= \\
& -M^{-1} S_{\text {total }}\left[\left|\bar{m}\left(\mathbf{r}, t_{n}\right)\right\rangle-\left|\bar{m}^{(e q)}\left(\mathbf{r}, t_{n}\right)\right\rangle\right],
\end{aligned}
$$

where $\left|\bar{f}\left(\mathbf{r}, t_{n}\right)\right\rangle, \quad\left|\bar{m}\left(\mathbf{r}, t_{n}\right)\right\rangle,\left|\bar{m}^{(e q)}\left(\mathbf{r}, t_{n}\right)\right\rangle$, and $S_{\text {total }}$ represent the distribution function vector, the moment vector, the equilibrium moment vector of the resolved scales and effective relaxation time matrix, respectively.

We note that the second-order moments of the distribution function, 
A.R. Rahmati et al. / JAFM, Vol. 7, No. 1, pp. 89-102, 2014.

$$
P_{i j}=\sum_{\alpha} e_{\alpha i} e_{\alpha j} f_{\alpha}=p \delta_{i j}+u_{i} u_{j}-\frac{1}{s_{x x}} 2 c_{S}^{2} S_{i j}
$$

where $e_{\alpha i}$ denotes the i-th Cartesian component of a discrete velocity $\mathbf{e}_{\alpha}$, are in fact related to the secondorder moments $p_{x x}$ and $p_{x y}$. In above formula, $s_{x x}$ is the relaxation rate for these second-order moments and $c_{s}$ is the sound speed. (In the setting of SRT-LB model, $s_{x x}=1 / \tau$ ) Therefore,

$S_{i j}=\frac{s_{x x}}{2 c_{s}^{2}}\left[p \delta_{i j}+u_{i} u_{j}-P_{i j}\right]=-\frac{s_{x x}}{2 c_{s}^{2}} Q_{i j}$.

The second-order monomials $\left\{e_{\alpha i} e_{\alpha j} \mid i, j \in\{x, y\}\right\}$ can be projected to the orthogonal basis vectors $\left\{\left|\phi_{\beta}\right\rangle \mid \beta=0,1, \ldots, N\right\}$, that is, the dual eigenvectors of $M$ :

$$
\begin{aligned}
& e_{\alpha x}^{2}=\frac{1}{6}\left[\left|\phi_{1}\right\rangle_{\alpha}+4\left|\phi_{0}\right\rangle_{\alpha}+3\left|\phi_{7}\right\rangle_{\alpha}\right], \\
& e_{\alpha y}^{2}=e_{\alpha x}^{2}-\left|\phi_{7}\right\rangle_{\alpha}, \\
& e_{\alpha x} e_{\alpha y}=\left|\phi_{8}\right\rangle_{\alpha} .
\end{aligned}
$$

Thus in this case the components of tensor $Q$ can be explicitly given in terms of the moments:

$$
\begin{aligned}
& Q_{x x}=P_{x x}-\left(p+u_{x} u_{x}\right), \\
& Q_{y y}=P_{y y}-\left(p+u_{y} u_{y}\right), \\
& Q_{x y}=P_{x y}-u_{x} u_{y}, \\
& Q_{x y}=Q_{x y}, \\
& P_{x x}=\frac{1}{6}\left[e+4 p+3 p_{x x}\right], \\
& P_{y y}=-p_{x x}+P_{x x}, \\
& P_{x y}=p_{x y} .
\end{aligned}
$$

The effect of the unresolved scales is modeled through an effective relaxation time scale $\tau_{t}$. Thus in Eq. (1) the total LES effective relaxation time should be $\tau_{\text {total }}=\tau_{t}+\tau_{0}$ where $\tau_{0}$ and $\tau_{t}$ are the relaxation times corresponding to the molecular viscosity $v_{0}$ and the turbulence or eddy viscosity $v_{t}$, respectively. Accordingly $v_{\text {total }}$ is given by (Krafczyk et al., 2003; Van Treeck et al., 2006):

$$
\begin{aligned}
& v_{\text {total }}=v_{0}+v_{t}= \\
& \frac{1}{3}\left(\tau_{\text {total }}-\frac{1}{2}\right) c^{2} \delta t=\frac{1}{3}\left(\tau_{0}+\tau_{t}-\frac{1}{2}\right) c^{2} \delta t, \\
& v_{t}=\frac{1}{3} \tau_{t} c^{2} \delta t,
\end{aligned}
$$

where $v_{t}$ depends on the sub-grid model used in the simulation. We use the Smagorinsky model and the mixed scale viscosity model for sub-grid closure.

In the Smagorinsky model, the eddy viscosity $v_{t}$ is calculated from the filtered strain rate tensor, and a filter length scale, $\bar{\Delta}$, as:

$$
\begin{aligned}
& v_{t}=\left(C_{S} \cdot \bar{\Delta}\right)^{2} \cdot|\bar{S}|, \\
& |\bar{S}|=\frac{|\bar{Q}|}{2 \rho_{0} c_{s}^{2} \tau_{\text {total }}}, \quad|\bar{Q}|=\sqrt{2 \bar{Q}_{l \gamma} \bar{Q}_{l \gamma}}
\end{aligned}
$$

where $\bar{S}$ and $\bar{Q}$ are the characteristic filtered rate of strain and the filtered mean momentum flux, respectively, and $C_{S}$ is the Smagorinsky constant.

The mixed scale viscosity model stems from a Smagorinsky model in which the local adaptation is achieved by taking into account the kinetic energy at the cut-off, $q_{c}$, explicitly. The sub-grid viscosity is then measured as (Sergent et al., 2006):

$v_{t}=0.07|\bar{S}|^{\frac{1}{2}}\left(q_{c}\right)^{\frac{1}{4}} \bar{\Delta}^{\frac{3}{2}}$

where $q_{c}$ stands for the kinetic energy at the cut-off, $q_{c}=\frac{1}{2} \bar{u}_{l}^{\prime} \bar{u}_{l}^{\prime} . \quad$ Following Bardina's similarity hypothesis, the velocity field at the cut-off, $\bar{u}_{l}^{\prime}$, can be estimated by filtering the resolved velocity field with a test filter coarser than the implicit one, $\tilde{\bar{\Delta}}>\bar{\Delta}$, and so $\vec{u}=\bar{u}-\tilde{\bar{u}}$.

The subgrid-scale dependency of this model ensures that it will adapt to the local state of the flow, and vanish in fully resolved regions of the flow and near the walls.

\subsection{Accomplishment on Non-Uniform Grid}

In standard lattice Boltzmann method, the grid is described as a regular lattice with identical spaces. But for high Rayleigh number flows, the thermal boundary layer is very thin. Hence it needs sizeable number of nodes which waste computational time and memory size. The Taylor series expansion and least square based lattice Boltzmann method (Niu et al., 2003) is based on the reality that density distribution function is a continuous function in the physical space and can be well defined in any mesh system. It is obtained from the standard LBM by using Taylor series expansion and optimized by the least squares technique. This method updates the density distribution functions at mesh points by an algebraic formulation and the pertinent coefficients are pre-calculated from the coordinates of 
mesh points. TLLB method allows mesh refinement near the walls.

Let us to consider a particle is initially at the grid point $(x, y, t)$. Along the i-direction, this particle will propagate to the position $\left(x+e_{\alpha x} \delta t, y+e_{\alpha y} \delta t, t+\delta t\right)$. For a uniform lattice, $\delta x=e_{\alpha x} \delta t, \delta y=e_{\alpha y} \delta t$. So, $\left(x+e_{\alpha x} \delta t, y+e_{\alpha y} \delta t\right)$ is on the grid point. In other words Eq. (1) can be used to update the density distribution functions exactly at the grid points. However, for a non-uniform grid, $\left(x+e_{\alpha x} \delta t, y+e_{\alpha y} \delta t\right)$ is usually not at the grid point $(x+\delta x, y+\delta y)$. In the numerical simulation, only the density distribution function at the mesh points for all the time levels are needed, so that the macroscopic properties such as the density, flow velocity and temperature can be evaluated at every mesh point. To get the density distribution function at the grid point $(x+\delta x, y+\delta y)$ and the time level $t+\delta t$, the Taylor series expansion in the spatial direction is applied.

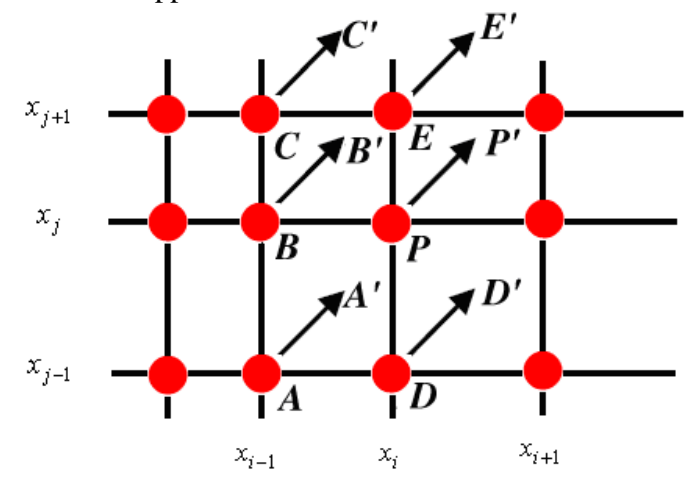

Fig. 2. Configuration of particle movement along the $\alpha$ direction.

As shown in Fig. 2, for simplicity, the point A represents the grid point $\left(x_{A}, y_{A}, t\right)$, point $A^{\prime}$ represents the position $\left(x_{A}+e_{\alpha x} \delta t, y_{A}+e_{\alpha y} \delta t, t+\delta t\right)$, and point $P$ represents the position $\left(x_{P}, y_{P}, t+\delta t\right)$ with $x_{p}=x_{A}+\delta x, y_{P}=y_{A}+\delta y$. So Eq. (1) gives

$f_{\alpha}\left(A^{\prime}, t+\delta t\right)=f_{\alpha}(A, t)+\frac{f_{\alpha}^{e q}(A, t)-f_{\alpha}(A, t)}{\tau}$.

For the general case, $A^{\prime}$ may not coincide with the mesh point $P$. We truncate the Taylor series expansion to the second order derivative terms. So $f_{\alpha}\left(A^{\prime}, t+\delta t\right)$ can be approximated by the corresponding function and its derivatives at the mesh point $P$ as

$$
\begin{aligned}
& f_{\alpha}\left(A^{\prime}, t+\delta t\right)=f_{\alpha}(P, t+\delta t)+ \\
& \Delta x_{A} \frac{\partial f_{\alpha}(A, t+\delta t)}{\partial x}+\Delta y_{A} \frac{\partial f_{\alpha}(A, t+\delta t)}{\partial y}+ \\
& \frac{1}{2}\left(\Delta x_{A}\right)^{2} \frac{\partial^{2} f_{\alpha}(A, t+\delta t)}{\partial x^{2}}+\frac{1}{2}\left(\Delta y_{A}\right)^{2} \frac{\partial^{2} f_{\alpha}(A, t+\delta t)}{\partial y^{2}}+ \\
& \left(\Delta x_{A} \Delta y_{A}\right) \frac{\partial^{2} f_{\alpha}(A, t+\delta t)}{\partial x \partial y}+O\left[\left(\Delta x_{A}\right)^{3},\left(\Delta y_{A}\right)^{3}\right]
\end{aligned}
$$

where $\Delta x_{A}=x_{A}+e_{\alpha x} \delta t-x_{P}, \quad \Delta y_{A}=y_{A}+e_{\alpha y} \delta t-y_{P}$.

For the two-dimensional case, this expansion involves six unknowns, that is, one density distribution function at the time level $t+\delta t$, two first-order derivatives, and three second-order derivatives. To solve for these unknowns, six equations are needed to close the system. This can be done by applying the second-order Taylor series expansion at six points: $P, A, B, C, D, E$. The following equation system can be obtained:

$f_{k}^{\prime}=\left\{s_{k}\right\}^{T}\{W\}=\sum_{i=1}^{6} s_{k l} W_{l}, \quad k=P, A, B, C, D, E$,

where

$f_{k}^{\prime}=$

$f_{\alpha}(x, y, t)+\frac{f_{\alpha}^{e q}(x, y, t)-f_{\alpha}(x, y, t)}{\tau}$,

$\left\{s_{k}\right\}^{T}=$

$\left\{1, \Delta x_{k}, \Delta y_{k},\left(\Delta x_{k}\right)^{2} / 2,\left(\Delta y_{k}\right)^{2} / 2, \Delta x_{k} \Delta y_{k}\right\}$,

$\{W\}=$

$\left\{f_{\alpha}, \partial f_{\alpha} / \partial x, \partial f_{\alpha} / \partial y, \partial^{2} f_{\alpha} / \partial x^{2}, \partial^{2} f_{\alpha} / \partial y^{2}, \partial^{2} f_{\alpha} / \partial x \partial y\right\}^{T}$.

Our aim is to find its first element $W_{1}=f_{\alpha}(P, t+\delta t)$. Eq. (18) can be put into the following matrix form

$[S]\{W\}=\left\{f^{\prime}\right\}$.

In practical applications, it was found that the matrix $[S]$ might be singular or ill-conditioned. To overcome this difficulty and make the method be more general, the least squares approach was introduced to optimize the approximation by Eq. (20). As a result, the equation system for $\{W\}$ becomes

$\{W\}=\left([S]^{T}[S]\right)^{-1}[S]^{T}\left\{f^{\prime}\right\}=[A]\left\{f^{\prime}\right\}$.

From Eq. (21), we can have

$$
\begin{aligned}
& f_{\alpha}\left(x_{0}, y_{0}, t+\delta t\right)=W_{1}=\sum_{k=1}^{M+1} a_{1, k} f_{k-1}^{\prime}, \\
& \left|f\left(\mathbf{r}_{p}, t+\delta t\right)\right\rangle=\sum_{k=1}^{M+1} a_{1, k}\left|f_{k-1}^{\prime}\right\rangle, \\
& \left|f_{k}^{\prime}\right\rangle=\left|f\left(\mathbf{r}_{k}, t_{n}\right)\right\rangle-M^{-1} S\left[\left|m\left(\mathbf{r}_{k}, t_{n}\right)\right\rangle-\left|m^{e q}\left(\mathbf{r}_{k}, t_{n}\right)\right\rangle\right] .
\end{aligned}
$$


where $a_{1, k}$ 's are the elements of the first row of the matrix A, which are defined by the coordinates of mesh points, the particle velocity and time step. They are not varied throughout the calculation. We can calculate once and store in advance, so little computational effort is introduced as compared with the standard LB method. On the other hand, Eq. (22) is nothing to do with the mesh structure. It needs only the information of coordinates of the mesh points. Thus, we can say that Eq. (22) can be consistently used for any kind of mesh structure.

\subsection{HTLB Scheme with a Sub-grid Model}

In the HTLB method (Van Treeck et al., 2006), the flow field is solved by the isothermal lattice Boltzmann equation while the advection-diffusion equation for temperature is solved separately by a finite difference scheme. The equation is used for solving the flow field is given by Eq. (8). The equation used for solving the temperature field is given by:

$$
\frac{\partial \bar{T}}{\partial t}+\bar{u} \frac{\partial \bar{T}}{\partial x}+\bar{v} \frac{\partial \bar{T}}{\partial y}=\left(\kappa+\kappa_{t}\right)\left(\frac{\partial^{2} \bar{T}}{\partial x^{2}}+\frac{\partial^{2} \bar{T}}{\partial y^{2}}\right),
$$

where $\kappa$ is the fluid thermal diffusivity and $\kappa_{t}$ is turbulent heat diffusivity. The energy equation is solved by a finite difference discritization on the non- uniform mesh. Both velocity components, $\bar{u}$ and $\bar{v}$ are obtained from Eq. (8). The relation between turbulent viscosity $v_{t}$ and the turbulent heat diffusivity $\kappa_{t}$ is given by the turbulent Prandtl number, i.e. $\operatorname{Pr}_{t}=v_{t} / \kappa_{t}$ which is assumed to be constant and equal to its theoretical value, i.e. 0.87 (Van Treeck et al., 2006).

\section{RESULTS AND DISCUSSION}

In the present study, an incompressible LBM solver is developed. At first a cavity driven flow is simulated to validate the code for simulation of isothermal incompressible flows on non-uniform grid. Then a Rayleigh-Bénard convective flow has been simulated at different Rayleigh numbers with Prantdl number of 0.71 .

\subsection{Lid-driven cavity flow}

Using the presented 2D TLLBM, numerical simulation of lid-driven flows in a cavity are considered for $R e=1000$, where $\operatorname{Re}=U L_{y} / v$ is the Reynolds number,

based on the lid velocity and the height of the cavity. The configuration of the problem is shown in Fig. 3. This problem has been widely used for validation and comparison purposes. Non-uniform mesh of $101 \times 101$ for $R e=1000$ is used. A typical non-uniform mesh in a square cavity for a $101 \times 101$ grid with stretch ratio of 2.54 , which is defined as the ratio of the maximum mesh spacing over the minimum mesh spacing, is shown in Fig. 4. The employment of non-uniform mesh is popular, particularly for simulation of the flow field at higher Reynolds numbers. This is because the boundary layer near the solid boundaries is thin. Hence, the mesh spacing near the wall should be very small to capture the thin boundary layer. The mesh spacing apart from the solid wall can be use relatively large. In this method, we can capture the thin boundary layer, and in the meantime, we can save the computational effort. Furthermore, the fluctuations which are created in the pressure domain at high Reynolds numbers can be reduced due to a decrease in the time step used for the simulation.

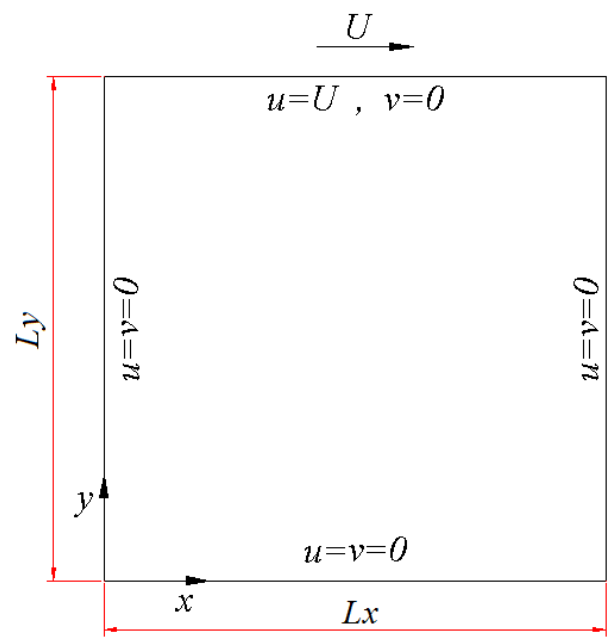

Fig. 3. 2D cavity flow.

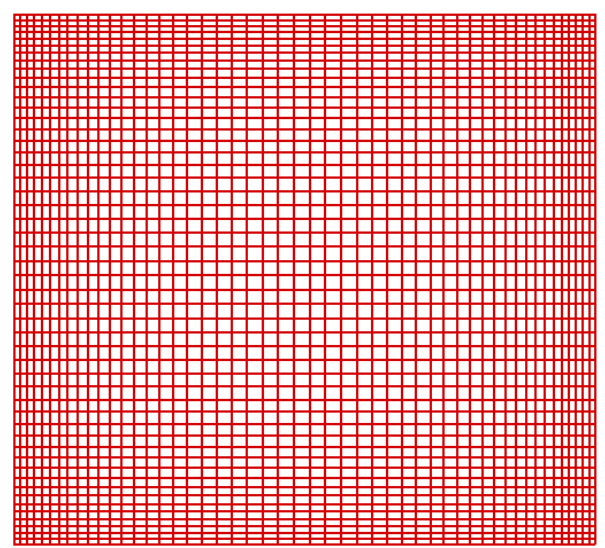

Fig. 4. The solution grid (a $101 \times 101$ non-uniform grid with stretch ratio of 2.54)

Initially, a constant pressure, $p=1 / 3$, is prescribed in the whole field, and the velocities in the interior of the cavity are set to zero. On the top, the $x$ component of the velocity is $U$, which is set to 0.19 , and the $y$ component of the velocity is zero. At the end of each time step, the density distribution function $f_{\alpha}$ on the moving wall is obtained by non-equilibrium boundary condition. The node wall bounce back boundary conditions are used on the other three stationary walls. 
All results which are putted on show in the current work are steady state solutions. In order to reach the steady state, a number of iterations are carried out. The criterion to steady sate is

$$
\sqrt{\frac{1}{N} \sum_{i=1}^{N}\left(u^{n+1}-u^{n}\right)^{2}+\left(v^{n+1}-v^{n}\right)^{2}} \leq 10^{-10},
$$

where $N$ is the total number of nodes in the solution domain; $\left(u^{n}, u^{n+1}\right)$ and $\left(v^{n}, v^{n+1}\right)$ are $\quad \mathrm{x}-\quad$ and $\mathrm{y}-$ component of the velocity for the old and new time levels, respectively.

Figures 5 and 6 present the $U$ and $V$ velocities along their respective centerline of the cavity flow at $R e=$ 1000 , respectively. As shown in those figures, the agreement between the Ghia's results (Ghia et al., 1982) and the present numerical results is very good.

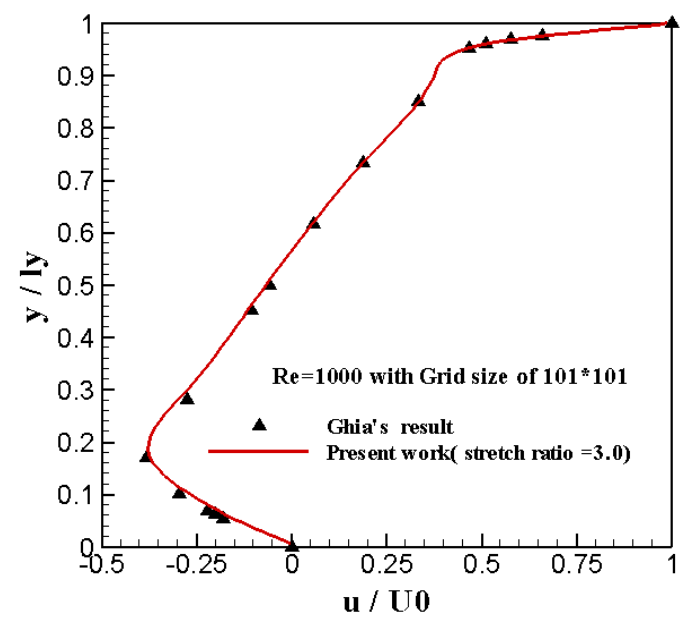

Fig. 5. The $x$-component velocity along vertical centerline cavity driven flow for $\mathrm{Re}=1000$.

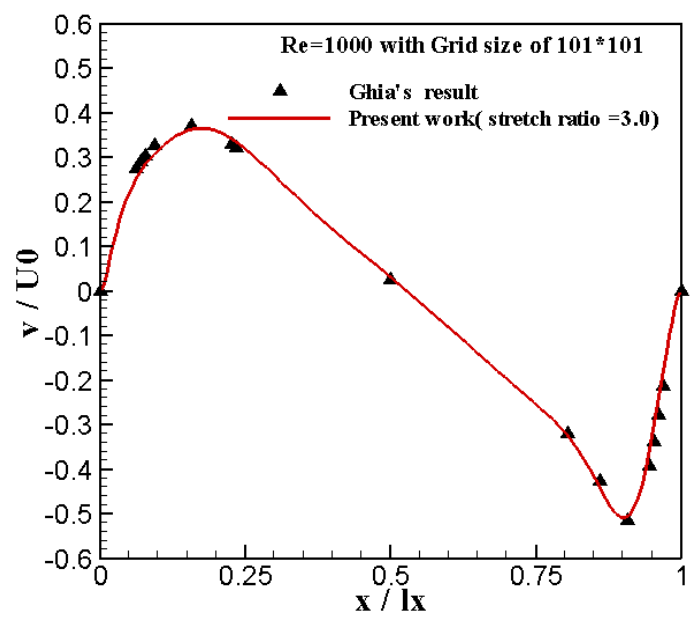

Fig. 6. The y-component velocity along horizontal centerline cavity driven flow for $\mathrm{Re}=1000$.

\subsection{Rayleigh-Bénard convection flow}

\subsubsection{Description of the problem}

Rayleigh-Bénard convection occurs in a wide variety of engineering applications. The flows are driven by the buoyancy effect due to the presence of gravity and density variations from low layer to another. When difference of temperature is lower, the flow is stationary. As difference of temperature is increased, the flow motion is regular and steady. As $R a$ is increased beyond the second critical Rayleigh number, fluctuation and instability of flows can be observed. In this natural convection problem, the initially static flow is heated from the bottom boundary, and a lower temperature is maintained at the upper wall. Additionally, a vertical gravitational force is applied to the y-direction of the computational domain. As the temperature difference between upper and bottom boundaries is increased, the stationary conduction state becomes unstable by any small disturbance.

According to linear stability theory, the critical wave number for Rayleigh-Bénard convection is $a_{c}=3.117$.

So that, the convective cells would be developed readily with an aspect ratio of $2 \pi / a_{c}=2.016$. An appropriate value of aspect ratio, $A R \equiv L_{x} / L_{y}=2$, for simulations can be determined.

The temperature difference between the hot and cold walls introduces a temperature gradient in a fluid, and the consequent density difference induces a fluid motion, that is, convection. In the simulation, the Boussinesq approximation is applied to the buoyancy force term.

$$
\rho \mathbf{G}=\rho \beta g T-T_{m} \mathbf{j}
$$

where $\beta$ is the thermal expansion coefficient, $g$ is the acceleration due to gravity, $T_{m}$ is the average temperature and $\mathbf{j}$ is the vertical direction opposite to that of gravity.

The dynamical similarity depends on two dimensionless parameters: the Prandtl number $P r$ and the Rayleigh number $R a$ defined as:

$\operatorname{Pr}=\frac{v}{\alpha}, R a=\frac{g \beta \Delta T L_{y}^{3}}{v \alpha}$,

where $L_{y}$ is the channel height, and $\Delta \mathrm{T}$ is the applied temperature difference between the bottom and top walls.

\subsubsection{Numerical Results}

\subsubsection{Laminar flow simulation}

Using the HT-LB model, we simulate Rayleigh-Bénard convection between two horizontal stationary walls for $R a=10^{4}$ with a $P r=0.71$ on different size of lattice (Fig. 7). To capture the thin boundary layer, a non-uniform mesh distribution is used (Fig. 8). Non-slip boundary 
conditions are implemented at the bottom and top boundaries by reversing the flow velocities in the ghost cell next to the simulation domain. The temperature along two opposite horizontal walls is maintained at constant temperatures 0 and 1 , respectively. Periodic boundary conditions are assigned for both the flow and the temperature fields along the side boundaries of the channel, see Fig. 7. A small perturbation in the form of a cosine wave with amplitude of $1 \times 10^{-3}$ is used to the pressure population. A linear distribution applied to the temperature field. Hence, at initial condition temperature is given by:

$$
T=T_{\text {cold }}-\frac{y-l y}{l y}\left(T_{\text {hot }}-T_{\text {cold }}\right) .
$$

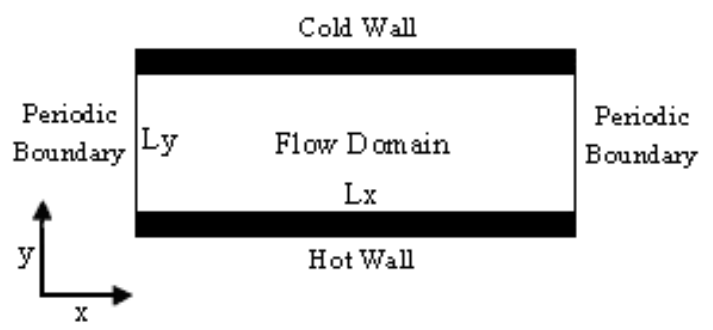

Fig. 7. Schematic of 2D enclosure Rayleigh- Bénard convection cell and boundary condition.

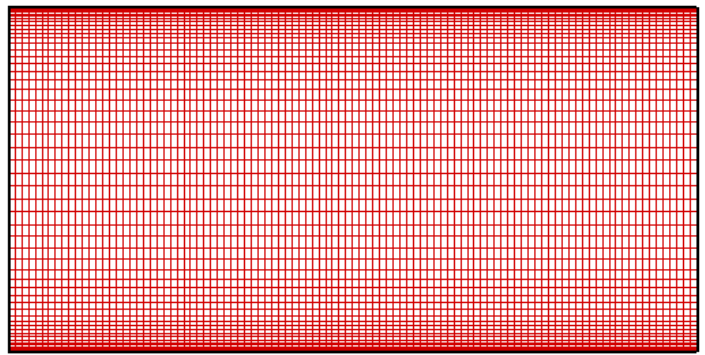

Fig. 8. The solution grid (a $101 \times 51$ non-uniform grid with stretch ratio of 3.15 ).

The Nusselt number can be calculated by the following equation:

$$
N u=1+\frac{\langle v T\rangle}{\alpha \Delta T / L_{y}},
$$

where $L_{y}$ is the vertical height of the channel, $\Delta T$ is fixed and denotes the temperature difference between the upper and bottom boundaries, $v$ is the flow velocity in the $y$-direction and $\langle$.$\rangle denotes the average over the$ whole flow domain. Table 1 presents the calculated Nusselt numbers using HT-LB model at $R a=10^{4}$, as well as the values obtained by the empirical formula (Chen and Doolen, 1998b; Shan, 1997), $N u=1.56\left(R a / R a_{c}\right)^{0.296}$ where $R a_{c}$ is the critical
Rayleigh number and is equal to $R a_{c}=1707.76$. From the presented results in Table 1, it can be seen that as the size of the grid is increased from $35 \times 17$ to $141 \times 71$, the discrepancy between the computed quantities is reduced from $14.0 \%$ to $0.65 \%$ for Nusselt number. This indicates that the employment of $101 \times 51$ grid can give accurate numerical results for $R a=10^{4}$.

Table 1 Grid dependence study for the RaylieghBénard convection at $R a=10^{4}$.

\begin{tabular}{|c|c|c|c|}
\hline $\begin{array}{c}R a \\
\text { no. }\end{array}$ & $\begin{array}{c}\text { Size of } \\
\text { mesh }\end{array}$ & $\begin{array}{c}\text { Present } \\
\text { work }\end{array}$ & $\begin{array}{c}\mathrm{Nu}= \\
1.56\left(\mathrm{Ra} / \mathrm{Ra} \mathrm{C}_{\mathrm{c}}\right)^{0.296}\end{array}$ \\
\hline $10^{4}$ & $35 \times 17$ & 2.264 & 2.632 \\
\hline $10^{4}$ & $71 \times 35$ & 2.423 & 2.632 \\
\hline $10^{4}$ & $101 \times 51$ & 2.609 & 2.632 \\
\hline $10^{4}$ & $141 \times 71$ & 2.615 & 2.632 \\
\hline
\end{tabular}

\subsubsection{Turbulent flow simulation}

In this study, the hybrid thermal lattice Boltzmann scheme is used along with the large-eddy approach to simulate turbulent convective flows. For the mass and momentum equations, a multi-relaxation-time LB technique is used while the heat equation is solved by a finite difference scheme. Furthermore, we applied the hybrid model by two subgrid scale models for both the fluid flow and the heat flux. Using HTLB-LES model, we simulate turbulent Rayleigh-Bénard convection between two horizontal stationary walls for $R a=10^{4}$ $10^{15}$ with $\operatorname{Pr}=0.71$ (Fig. 7). To capture the thin boundary layer, a non-uniform mesh distribution is used. As shown in Fig. 8, non-uniform meshes of $101 \times 51$ for $R a=10^{4}, 201 \times 101$ for $R a=10^{5}, 301 \times 151$ for $R a=10^{6}, 361 \times 181$ for $R a=10^{7}$, and $401 \times 201$ for $R a=10^{8}$ - $10^{15}$, respectively. The stretch ratio is 3.15 for all cases. No-slip boundary conditions are performed at the bottom and top walls by using the mid-wall bounceback scheme in LB simulation. The temperatures along two opposite horizontal walls are maintained constant, 0 and 1 . Periodic boundary conditions are assigned for both the flow and the temperature fields at the inlet and outlet of the channel, see Fig. 7, Figs. 9 and 10 show streamlines and temperature contours at different $R a$ numbers. As shown in Figs. 9 and 10, the hot fluid near the bottom wall moves upward and intensifies the temperature in the central part of the flow domain, while the cold fluid close to the top wall streams downward and reduces the temperature near the side boundaries. When the Rayleigh number increases, two tendencies were perceived for the temperature contour distributions: 1) an increase in the temperature gradients close to the top and bottom boundaries and 2) intensified mixing of the hot and cold fluids. Both inclinations enhance the heat transfer in the flow domain. 
A.R. Rahmati et al. / JAFM, Vol. 7, No. 1, pp. 89-102, 2014.

(a) $\mathrm{Ra}=10^{4}$

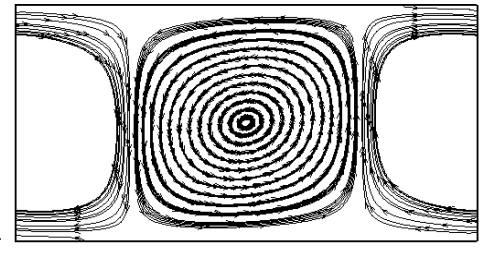

(b) $\mathrm{Ra}=10$

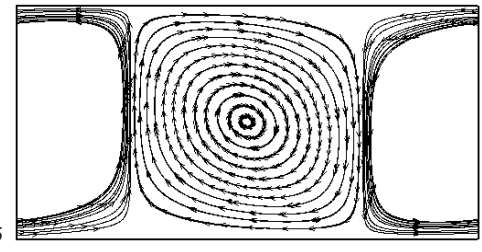

(c) $\mathrm{Ra}=10^{6}$

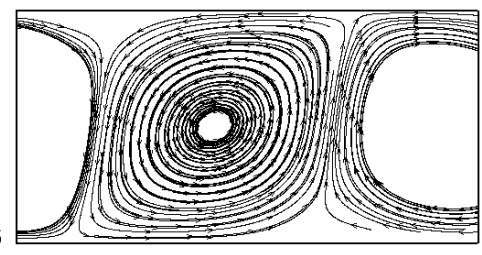

(d) $\mathrm{Ra}=10^{7}$

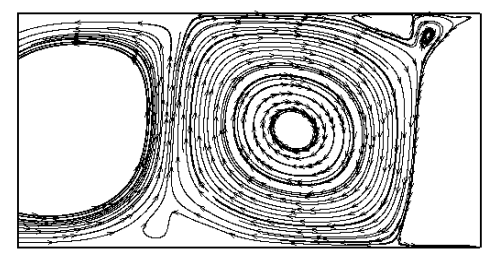

(e) $\mathrm{Ra}=10^{8}$

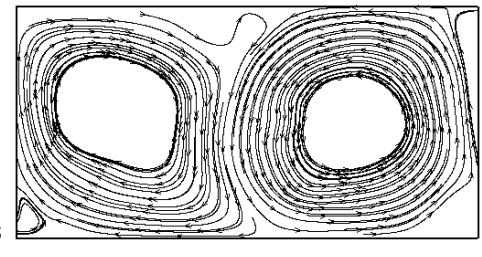

(f) $\mathrm{Ra}=10^{9}$

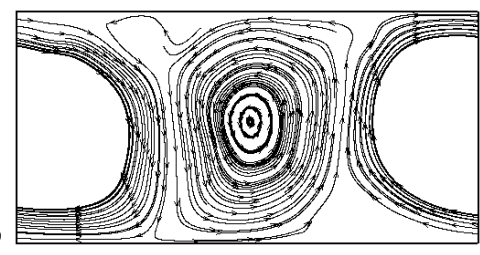

(g) $\mathrm{Ra}=10^{10}$

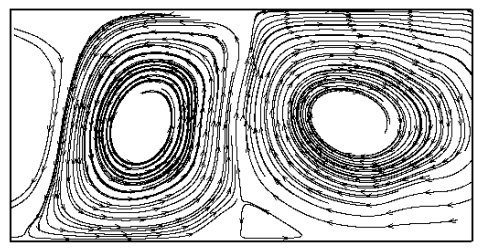

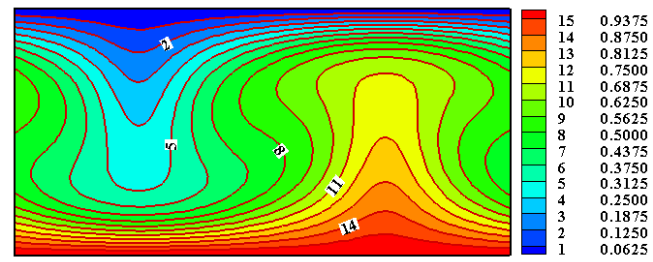
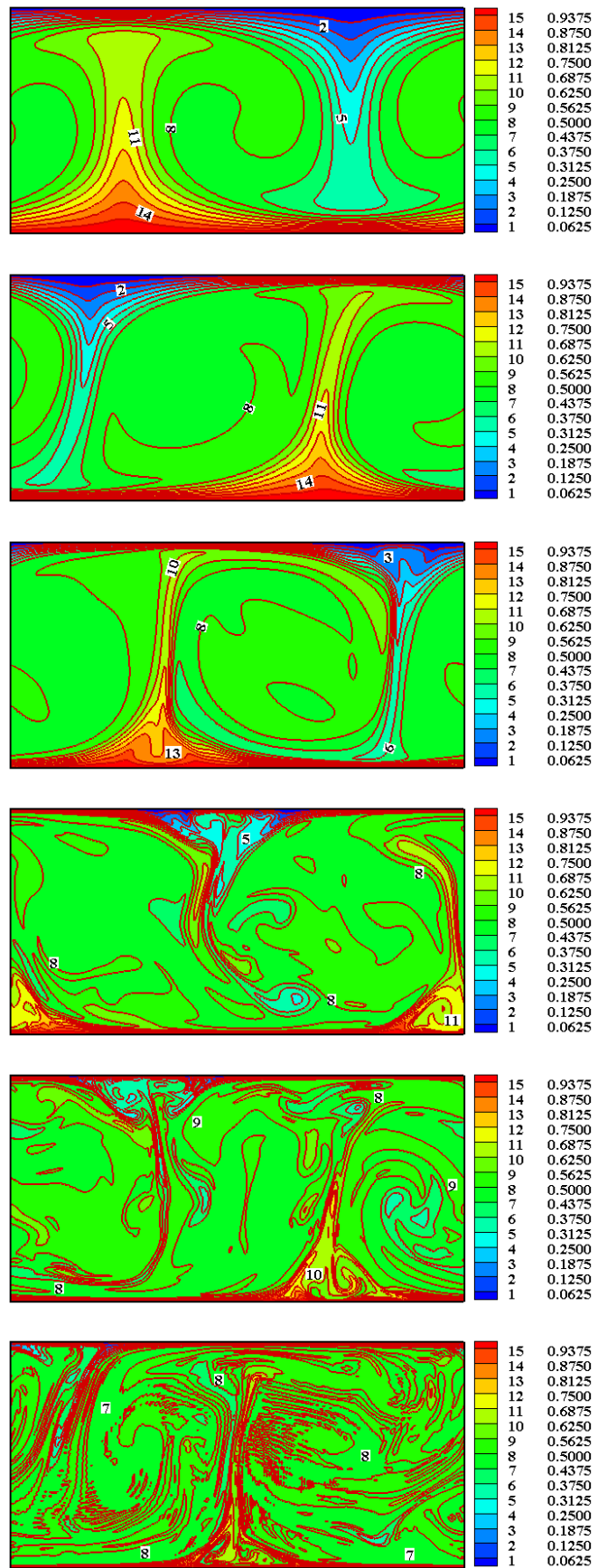
(h) $\mathrm{Ra}=10^{15}$
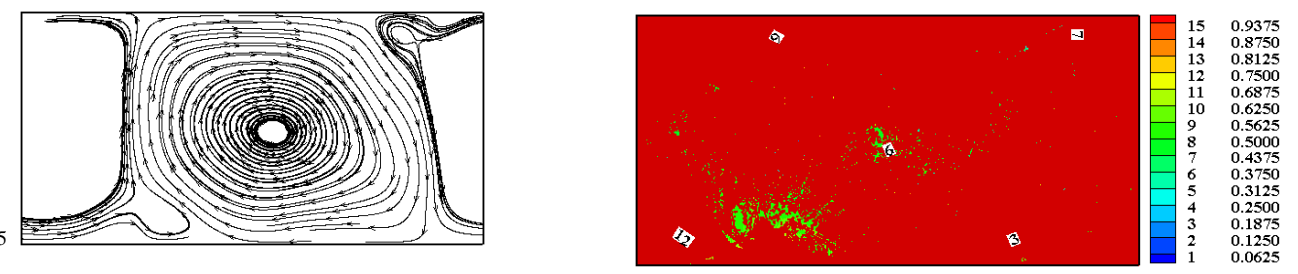

Fig. 9. Streamlines (left side) and temperature contours (right side) obtained by Smagorinsky model for RayleighBénard convection at the 8000000 -th time step for different Rayleigh numbers.

(a) $\mathrm{Ra}=10^{4}$

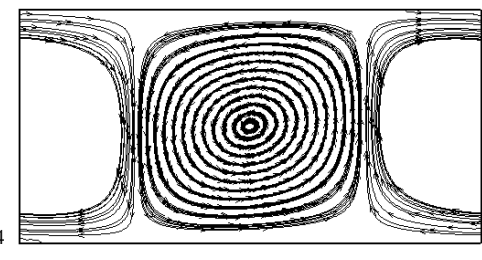

(b) $\mathrm{Ra}=10^{5}$

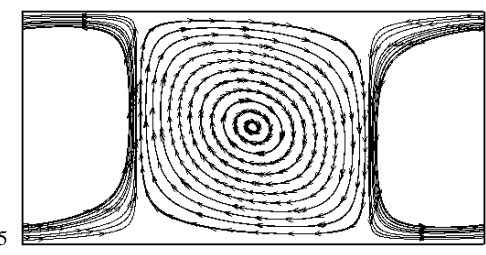

(c) $\mathrm{Ra}=10^{6}$

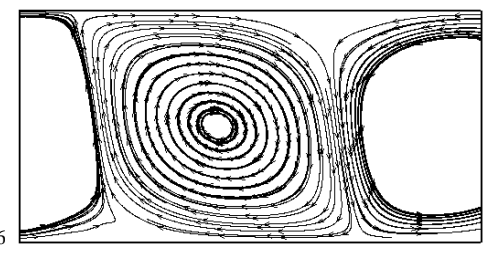

(d) $\mathrm{Ra}=10^{7}$

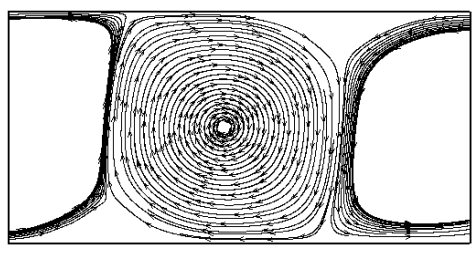

(e) $\mathrm{Ra}=10^{8}$

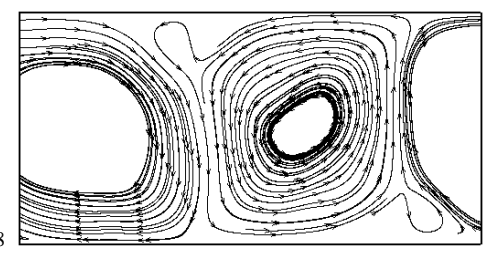

(f) $\mathrm{Ra}=10^{9}$

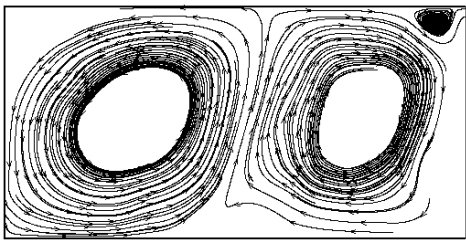

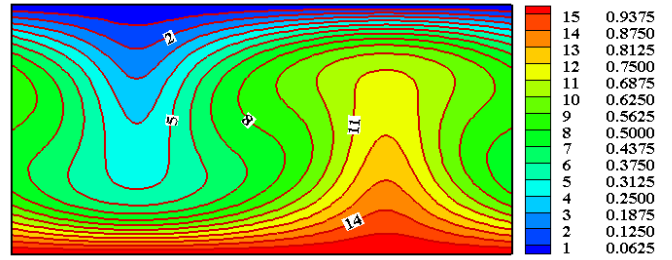
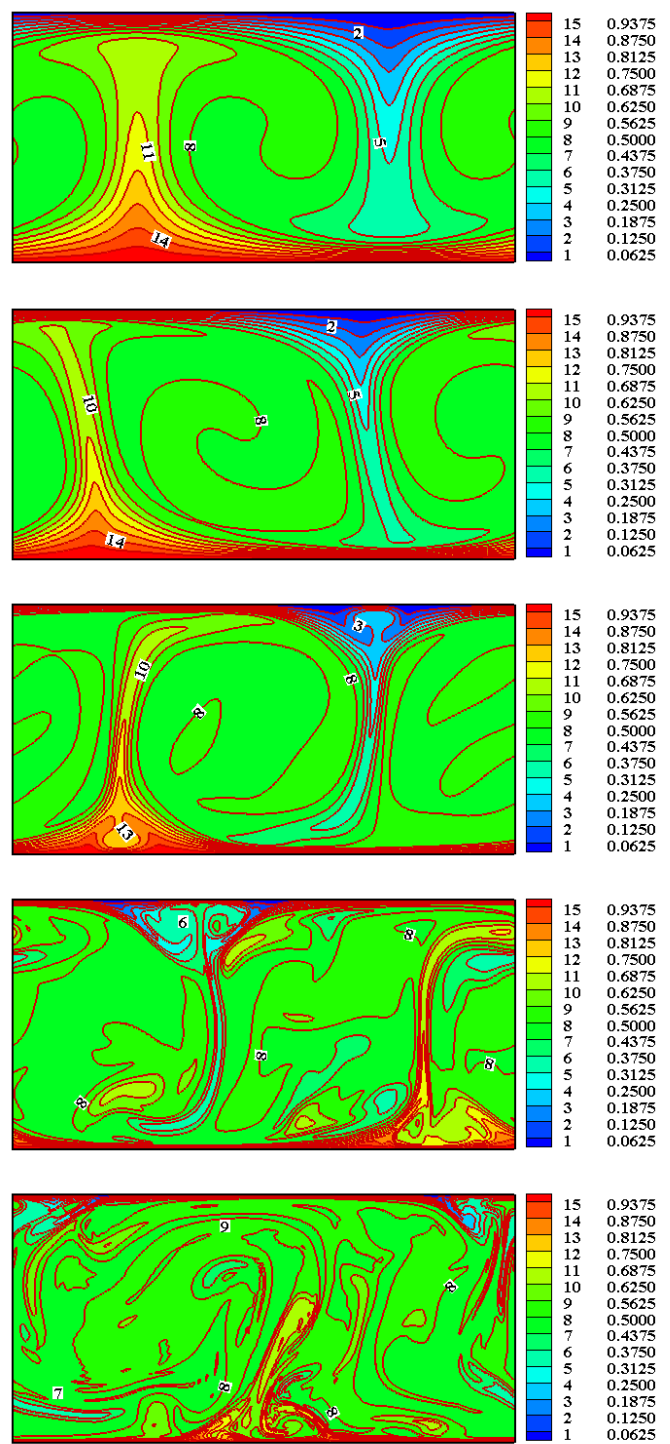
(g) $\mathrm{Ra}=10^{10}$

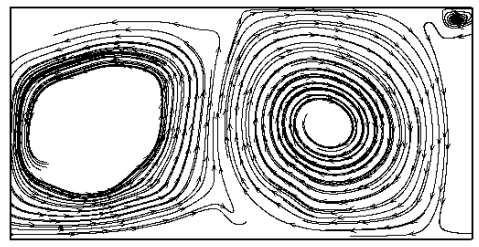

(h) $\mathrm{Ra}=10^{15}$

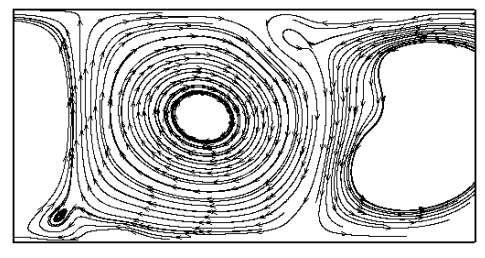

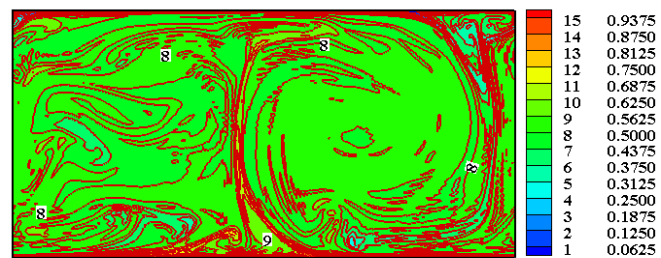

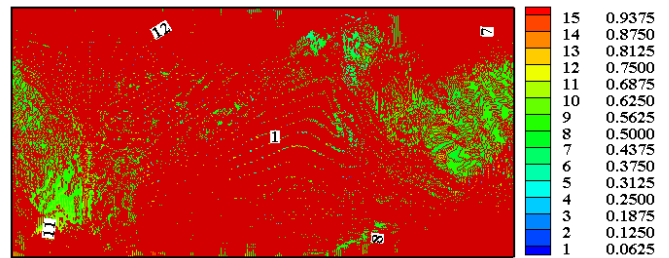

Fig. 10. Streamlines (left side) and temperature contours (right side) obtained by mixed scale model for RayleighBénard convection at the 8000000-th time step for different Rayleigh numbers.

Time evolution of the $\mathrm{x}$ and $\mathrm{y}$ components of the velocity vector, and the temperature obtained by both Smagorinsky model (left side) and mixed scale model (right side) at the mid of flow domain for $R a=10^{10}$ is shown in Fig. 11. Furthermore, Fig. 12 presents power spectral density of the kinetic energy fluctuations at the mid of flow domain obtained by Smagorinsky model (left side) and mixed scale model (right side) for $R a=10^{10}$. As shown in Fig. 12, slope of inertial subrange obtained by both Smagorinsky model and mixed scale model is in a better agreement with -3 power law compared to $-5 / 3$ power law (Frost and Moulden, 1977).

An important feature is the total heat transport that is described in terms of the Nusselt number $N u=1+\langle\bar{v} \bar{T}\rangle L_{y} / \alpha \Delta T$. Here $\bar{v}$ is the mean vertical velocity, and \langle\rangle denotes the average over the whole domain. Table 2 presents the calculated Nusselt number at $R a=10^{4}-10^{10}$, as well as DNS results obtained by Kerr (1996). It is observed that our results are in good agreement with the empirical data for lower Rayleigh number (up to $10^{4}$ ). As the Rayleigh number increases the computed Nusselt number is under predicted compared to the Nusselt number calculated by Kerr (1996). The under-prediction of the Nusselt number may be due to insufficient spatial resolution. Furthermore, it is observed that the results obtained using the mixed scale model, are in a better agreement with those reported in Ref. (Kerr, 1996), compared to the Smagorinsky model results.

\section{CONCLUSION}

In this work, a new LBM solver is proposed to simulate turbulent convection flows problems at high Rayleigh numbers. In order to illustrate the capability of the code to simulate isothermal flows, a two-dimensional cavity driven flow has first been simulated at Reynolds number of 1000 . Then its capability to solve the heat transfer problem in the case of the Rayliegh-Bénard convective flow up to $R a=10^{15}$ is shown. In the code, a MRT-LB model in conjunction with both Smagorinsky and mixed scale viscosity sub-grid models is used to simulate a turbulent Rayleigh-Bénard convection at different Rayleigh numbers. The combination of the MRT-LB method with the subgrid model allows us to simulate the turbulent convection flows up to a Rayleigh number of $10^{15}$. In the proposed code, a spacefiltered density distribution function is defined. In Smagorinsky subgrid model, the energy dissipation caused by the interaction between resolved and unresolved scales is included. In current method the local strain intensity stress is calculated locally at each time step using the non-equilibrium density distribution functions. In order to get proper near-wall behaviour or to simulate flows which are not fully turbulent, the subgrid model should be able to adjust itself to locally inhomogeneous flow. The mixed scale viscosity model ensures that it will adapt to the local state of the flow, and vanish in fully resolved regions of the flow and near the walls. Results show that the proposed method produce reasonably accurate results at low Rayleigh numbers and stable results at high Rayleigh numbers. Besides, it is observed that the results obtained using the mixed scale model, are in a better agreement with existing CFD data, compared to the Smagorinsky model results. 
(a) $u$
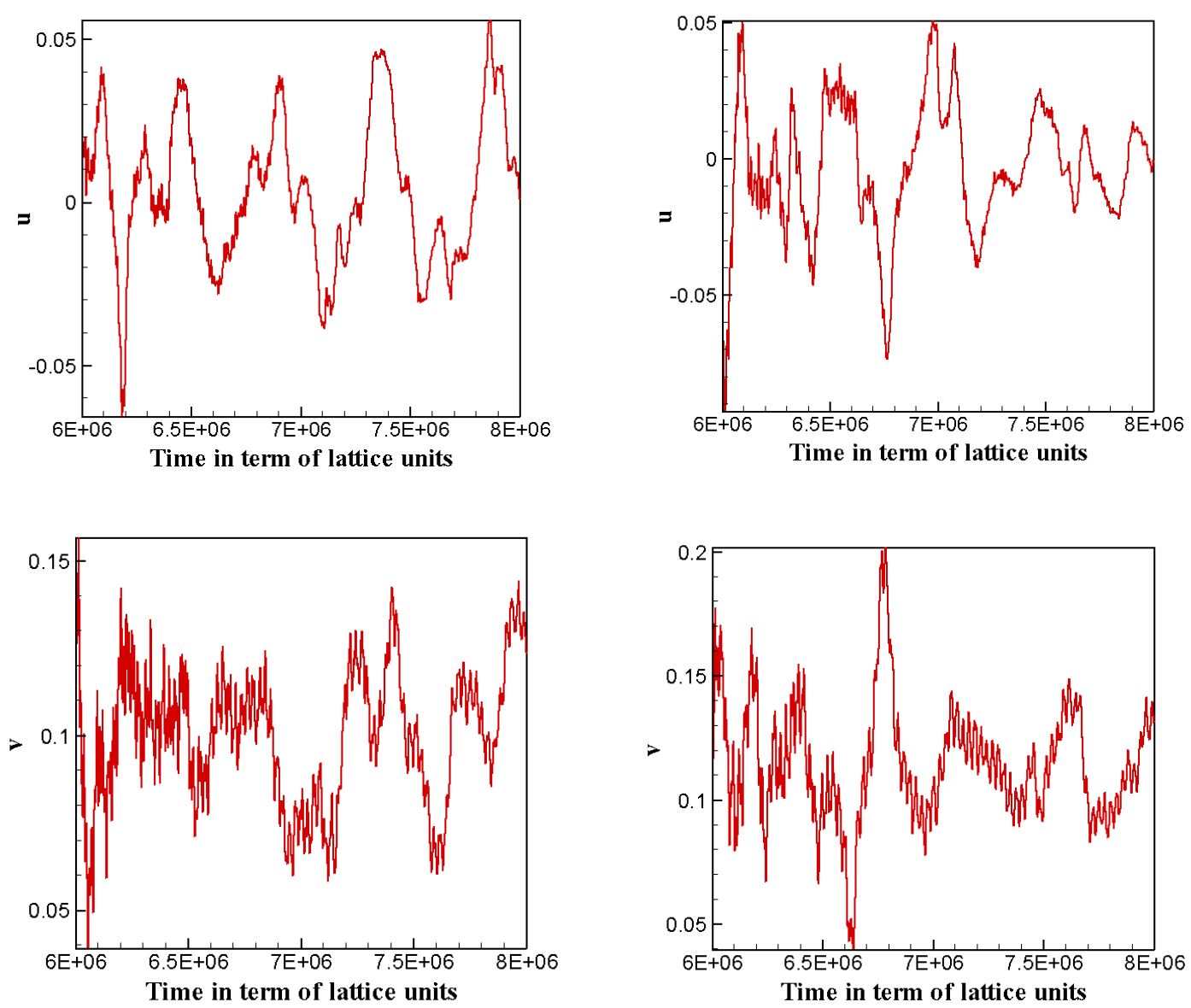

(b) $v$
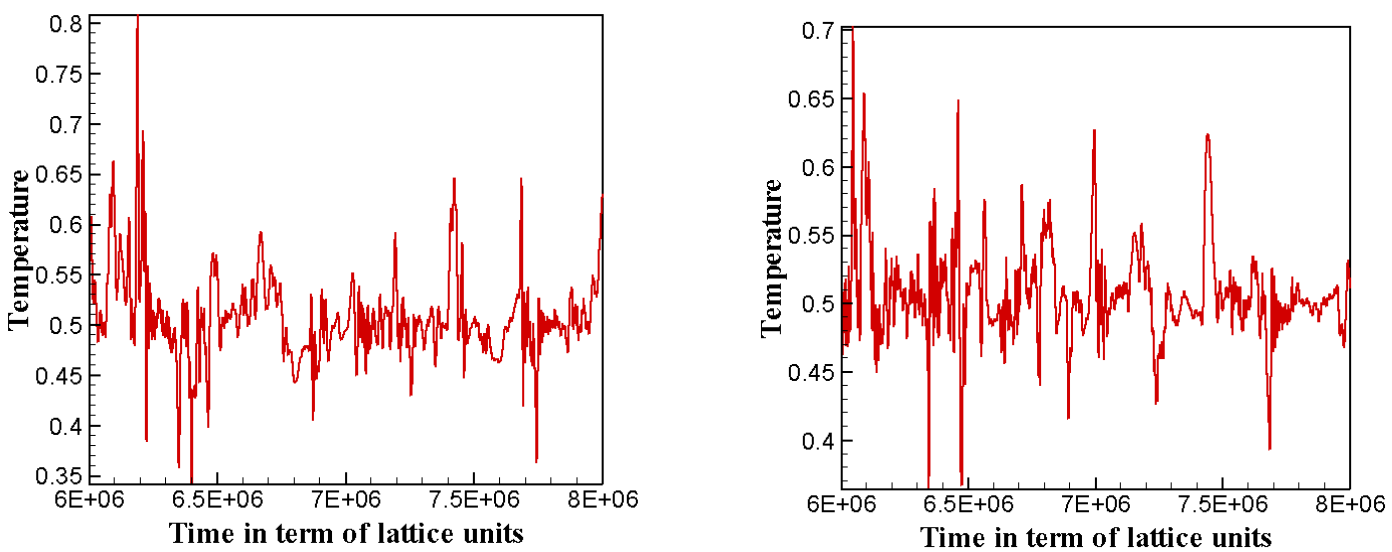

Fig. 11. Time evolution of the $\mathrm{x}$ and $\mathrm{y}$ components of the velocity vector, and the temperature obtained by both Smagorinsky model (left side) and mixed scale model (right side) at the mid of flow domain for $R a=10^{10}$. 

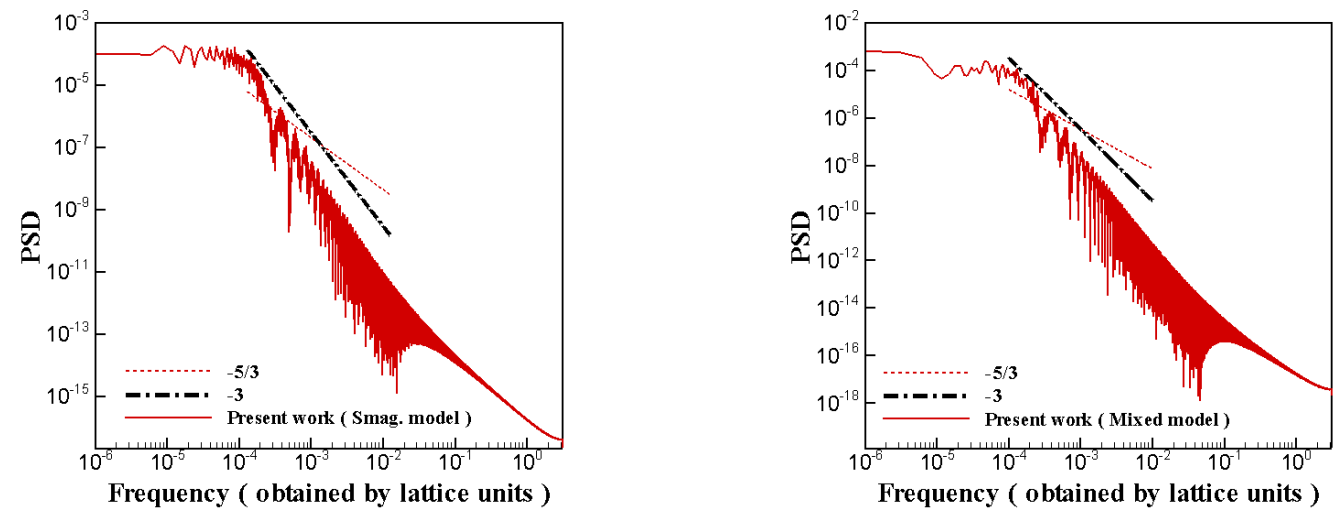

Fig. 12. Power spectral density of the kinetic energy fluctuations obtained by Smagorinsky model (left side) and mixed scale model (right side) at the mid of flow domain for $R a=10^{10}$.

Table 2 Nusselt numbers computed for Rayleigh-Bénard convection for different $R a$.

\begin{tabular}{|c|c|c|c|c|}
\hline \multirow{2}{*}{$R a$ no. } & \multirow{2}{*}{ Size of mesh } & \multicolumn{2}{|c|}{ Present work } & \multirow{2}{*}{ Kerr $\square$ s results } \\
\cline { 3 - 5 } & & Smagorinsky model & Mixed scale model & \\
\hline $10^{4}$ & $101 \times 51$ & 2.6690 & 2.6687 & 2.363 \\
\hline $10^{5}$ & $201 \times 101$ & 4.971 & 4.968 & 4.462 \\
\hline $10^{6}$ & $301 \times 151$ & 8.169 & 8.3535 & 8.424 \\
\hline $10^{7}$ & $361 \times 181$ & 13.729 & 14.016 & 15.904 \\
\hline $10^{8}$ & $401 \times 201$ & 24.252 & 22.611 & - \\
\hline $10^{9}$ & $401 \times 201$ & 38.237 & 33.302 & - \\
\hline $10^{10}$ & $401 \times 201$ & 72.93 & 71.76 & - \\
\hline
\end{tabular}

\section{ACKNOWLEDGMENT}

The authors are grateful to University of Kashan for supporting this work by Grant No (158567/2).

\section{REFERENCES}

Chen, S. and G., Doolen (1998). Lattice Boltzmann method for fluid flows, Annu. Rev. Fluid Mech., 30, 329-364.

Chen, S. and G.D., Doolen (1998). A Novel Thermal Model for the Lattice Boltzmann Method in Incompressible Limit, J. Comput. Phys., 146, 282300 .

Du, R., B., Shi, and X., Chen (2006). Multi-relaxationtime lattice Boltzmann model for incompressible flow, Phys. Lett. A, 359(6), 564-572.
Frost, W., and T.H., Moulden (1977) Handbook of turbulence. Volume 1 - Fundamentals and applications, Plenum Press, New York.

Ghia, U., K.N., Ghia, and C., Shin (1982). High Reynolds Solutions for Incompressible Flow Using the Navier-Stokes Equations and a Multi-grid Method, Journal of Computational Physics, 45, 387-411.

Kadanoff, L. P., 2001. Turbulent heat flow: Structures and scaling. Phys. Today, 54 (8), 34-39.

Kerr, R. M., (1996). Rayleigh number scaling in numerical convection, J. Fluid Mech., 310, 139179.

Lallemand, P., and L.S. Luo (2003). Theory of the lattice Boltzmann method: Acoustic and thermal 
A.R. Rahmati et al. / JAFM, Vol. 7, No. 1, pp. 89-102, 2014.

properties in two and three dimensions, Phys. Rev. E, 68(3), 036706(1)-036706(25).

Niu, X. D., Y.T., Chew, and C., Shu (2003). Simulation of flows around an impulsively started circular cylinder by Taylor series expansion- and least squares-based lattice Boltzmann method, $J$. Comput. Phys.,188, 176-193.

Nwatchok, S.A.A., C.M., Biouele, and H.E. Fouda (2010). Lattice Boltzmann simulation of the twodimensional Poiseuille-Rayleigh-Benard flows instability, Int. J. Phys. Sci., 5(7), 984-991.

Kao, P. H., and R.J., Yang (2007). Simulating oscillatory flows in Rayleigh-Bénard convection using the lattice Boltzmann method, Int. J. Heat and Mass Transfer, 50 (17/18), 3315-3328.

Krafczyk, M., J., Tölke, and L.S., Luo (2003). Largeeddy simulations with a multiple-relaxation-time LBE model, Int. J. Mod. Phys. B, 17, 33-39.

Rahmati, A. R., and M., Ashrafizaadeh (2010). Stability Improvement of Lattice Boltzmann Method for Simulation of High Rayleigh Turbulent Thermal Flows, PhD Thesis, Isfahan University of Technology, Isfahan, Iran.
Shan, X., (1997). Simulation of Rayleigh-Bénard convection using lattice Boltzmann method, Phys. Rev. E, 55(3), 2780-2788.

Sergent, A., P., Joubert, and P., Le Quéré (2006). Large eddy simulation of turbulent thermal convection using a Mixed Scale Diffusivity Model, Prog. Comput. Fluid Dynam., 6(1/2/3), 40-49.

Siggia, E. D., (1994). High Rayleigh number convection. Annu. Rev. Fluid Mech., 26, 137-168.

Sreenivasan, K. R., and R.J., Donnelly (2001). Estimated Rayleigh numbers in naturally occurring processes. Adv. Appl. Mech., 37, 239.

Teixeira C., H., Chen, and DM. Freed (2000). Multispeed thermal lattice Boltzmann method stabilization via equilibrium under-relaxation, Comput. Phys. Comm., 129(1/3), 207-226.

Van Treeck, C., E. Rank, and M., Krafczyk (2006). Extension of a hybrid thermal LBE scheme for large-eddy simulations of turbulent convective flows, Comput. \& Fluids, 35(8/9), 863-871. 